'Departamento de Neurología y Medicina Interna, Clínica Las Condes. Santiago, Chile.

2Departamento de Neurología \& Neurocirugía, Hospital Clínico Universidad de Chile. Santiago, Chile.

Recibido el 10 de agosto de 2020, aceptado el 16 de noviembre de 2020

Los autores declaran no tener conflictos de interés. Trabajo no recibió financiamiento.

Correspondencia a:

Dr. Mario Campero Lo Fontecilla 441, Las Condes, Santiago, Chile. mcampero@clc.cl

\section{La brucelosis en el diagnóstico diferencial de las meningitis asépticas: a propósito de un caso}

\author{
MARIO CAMPERO ${ }^{1,2}$, RICARDO ESPINOZA $^{1}$, CARLOS SILVA $^{2}$
}

\begin{abstract}
Aseptic meningitis represents a diagnostic challenge for the clinician. Cytological and chemical parameters are key in the differential diagnosis. Hypoglycorrhachia is a strong predictor of a bacterial origin for aseptic meningitis. We report a 44-year-old male with a history of recurrent febrile headaches admitted with fever and delirium. The initial cerebrospinal fluid (CSF) analysis showed low glucose levels. Magnetic resonance imaging did not show abnormalities. The patient was discharged but was admitted again three weeks later with fever, headache and a stiff neck. The CSF was inflammatory with low glucose levels. Serology for brucellosis was positive. The patient was treated with ceftriaxone and rifampicin with a good clinical response.
\end{abstract}

(Rev Med Chile 2020; 148: 1844-1847)

Key words: Brucellosis; Headache; Meningitis, Aseptic.
L a meningitis aséptica (MA) es un proceso inflamatorio que afecta a las envolturas del sistema nervioso central $(\mathrm{SNC})^{1-3}$. El concepto "aséptico" está referido a la ausencia de microorganismos en los cultivos habituales para agentes bacterianos en el líquido cefalorraquídeo $(\mathrm{LCR})^{1-3}$. Un paciente con una MA constituye un desafío diagnóstico y el estudio debe incluir la búsqueda de bacterias, virus, hongos y pará$\operatorname{sitos}^{1,3}$. Además, deben considerarse las MA no infecciosas como son las neoplasias que infiltran las meninges ${ }^{1-6}$.

Las características fisicoquímicas y citológicas del LCR constituyen el primer elemento que permiten orientar a una posible causa. El valor de la glucosa en el LCR tiene un alto valor predictivo en el diagnóstico de las MA bacterianas $y$, particularmente, las piogénicas ${ }^{3,4}$.

La reacción de polimerasa en cadena (Polymerase Chain Reaction o PCR) permite la detección de un amplio número de microrganismos ${ }^{3}$. Además, se disponen de cultivos corrientes y especiales ${ }^{3}$.
El estudio citológico y la citometría de flujo son poderosas herramientas para el diagnóstico ${ }^{3}$.

En este artículo presentamos a un enfermo con una MA causada por una brucelosis con el antecedente de un viaje al extranjero, caracterizada por un síndrome febril prolongado y recurrente. Asimismo, destacamos la utilidad de la hipoglucorraquia en el diagnóstico diferencial.

\section{Caso clínico}

Hombre sano de 44 años con el antecedente de un viaje a México un mes antes del inicio de sus síntomas. En forma insidiosa inició una tos irritativa y una cefalea frontotemporal. La radiografía de tórax fue normal. Se asoció una baja de peso estimada en cuatro kg, sin anorexia. A pesar de haber recibido ampicilina en forma ambulatoria. Un mes antes de su ingreso se agregó sensación febril, presencia de una cefalea frontotemporal, incremento de la tos y fiebre oscilante. 
Ingresó por un síndrome confusional agitado, con una temperatura axilar de $38,4{ }^{\circ} \mathrm{C}$ y rigidez de nuca. Se realizó un escáner de cerebro, sin anomalías. Se inició, empíricamente, ceftriaxona, ampicilina y aciclovir con la sospecha de una meningitis bacteriana o herpética. Se internó en la Unidad de Paciente Crítico.

El estudio de LCR mostró una proteinorraquia de $75 \mathrm{mg} / \mathrm{dL}$, una glucorraquia de $27 \mathrm{mg} / \mathrm{dL}$ (glicemia concomitante $126 \mathrm{mg} / \mathrm{dL}$ ) y 78 glóbulos blancos, 92\% mononucleares. La serología para VIH, citomegalovirus, virus de Epstein Barr, criptococo, Haemophilus, meningococo y neumococo fueron negativas. Los cultivos corrientes, hongos y Koch resultaron repetidamente negativos. El estudio de resonancia magnética de encéfalo y columna completa no mostraron captación del contraste meníngeo ni radicular ni lesiones focales del SNC.

Los estudios generales no dieron cuenta de un fenómeno inflamatorio, con una VHS, proteína $\mathrm{C}$ reactiva y procalcitonina dentro de rangos normales.

El paciente evolucionó sin compromiso de conciencia, tos y cefalea asociada a alzas febriles hasta $39^{\circ} \mathrm{C}$. Los hemocultivos y el ecocardiograma fueron negativos. Los estudios de Quantiferon ${ }^{\circledR}$ para TBC, serología para enfermedad de Lyme y VDRL fueron negativos o no reactivos. Una nueva punción lumbar (PL) no mostró células neoplásicas en el frotis de LCR. Un PET (F18-FDG) de cuerpo completo no evidenció zonas hipermetabólicas. Gradualmente, la fiebre comenzó a descender y la cefalea a ceder. Al cabo de diez días se decidió el alta, manteniendo la ampicilina i.v. ante una posible meningitis por Listeria monocytogenes.

Durante el alta, se mantuvo afebril durante tres semanas, con una mínima cefalea temporal y tos que fue en regresión. Al final de este periodo, reinició su síndrome febril, con temperaturas axilares de hasta $39,5^{\circ} \mathrm{C}$, una intensa cefalea y un examen neurológico normal, salvo la rigidez de nuca. La PL mostró un LCR inflamatorio con $232 \mathrm{mg} / \mathrm{dL}$ de proteínas, 75 células (95\% mononucleares) y 32 $\mathrm{mg} / \mathrm{dL}$ de glucorraquia (glicemia de $134 \mathrm{mg} / \mathrm{ml}$ ).

Considerando el viaje al extranjero, se realizó una prueba de aglutinación para brucela siendo positiva (abortus/melitensis) en diluciones de 1:320 y una prueba de ELISA positiva IgG e IgM. Inmediatamente, se inició ceftriaxona i.v. (2 g cada $12 \mathrm{~h}$ ) y rifampicina oral ( $600 \mathrm{mg} /$ día $)$. El paciente evolucionó sin nuevas alzas febriles y con una disminución de su cefalea. No hubo recaída sintomática en los controles posteriores. El paciente dio su consentimiento escrito de manera informada para fines de esta publicación.

\section{Discusión}

Las MA no son de fácil diagnóstico ${ }^{2,3}$. Afortunadamente, hoy disponemos de exámenes que permiten detectar partículas de $\mathrm{ADN}$ de diversos microrganismos, además de la determinación de anticuerpos contra estos ${ }^{3}$. La precocidad de la identificación de un agente causal depende de la gravedad del cuadro clínico. Este factor temporal en el diagnóstico de una MA es muy relevante pues determina el inicio y la duración de la terapia antimicrobiana específica ${ }^{3}$. De los aspectos del LCR, la hipoglucorraquia es uno de los hallazgos más robusto para plantear una etiología bacteriana $^{2}$. Un valor de $18 \mathrm{mg} / \mathrm{dL}$ tiene una muy alta probabilidad que corresponda a una meningitis bacteriana piogénica ${ }^{4}$. Sin embargo, se ha establecido que una cifra entre 20 y $25 \mathrm{mg} / \mathrm{dL}^{3,4}$ es más sensible para las MA. Por otra parte, a más baja la glucosa en el LCR, mayor es la mortalidad ${ }^{3,4}$. La mayor parte de la glucosa en el LCR llega por transportadores activos de los plexos coroideos, capilares ventriculares y subaracnoideos ${ }^{3}$. Solo una pequeña fracción entra por difusión simple ${ }^{3}$. El valor normal corresponde a $60 \%$ de la glicemia contemporánea a la PL, concepto clave para ponderar "la glucorraquia real"3,4. La hipoglucorraquia se explica por el consumo de la glucosa por las células inflamatorias y microorganismos ${ }^{3,4}$. No obstante, el mecanismo más importante es por un efecto sobre los transportadores activos de la glucosa ${ }^{3,4}$. Prueba de ello es la existencia de meningitis virales que pueden tener hipoglucorraquia ${ }^{3}$. La Tabla 1 muestra la orientación etiológica en función de los valores de la glucosa en el $\mathrm{LCR}^{1,4}$.

En nuestro paciente, la sospecha de una meningitis se confirmó con la elevación de las proteínas y células en el LCR. Aun teniendo repetidamente una hipoglucorraquia, ningún agente infeccioso convencional fue encontrado. Por otra parte, aquellas causas de meningitis infecciosa como la tuberculosis, la sífilis, la neuroborreliosis y el VIH, entre otras, fueron descartadas con los exámenes correspondientes. Destaca que en Chile no han sido descrito casos endémicos de enfermedad 
Tabla 1. Hipoglucorraquia: orientación etiológica

\begin{tabular}{|ll|}
\hline Glucorraquia menor A $\mathbf{2 0 - 2 5} \mathbf{~ m g / d L}$ & Glucorraquia mayor a $\mathbf{2 0 - 2 5} \mathbf{~ m g / d L}$ \\
Meningitis bacterianas piogénicas & Mycoplasma y Ureaplasma \\
- Neisseria meningitidis & $-\quad$ Mycoplasma pneumoniae \\
- Streptococcus pneumoniae & \\
- Staphylococcus aureus & \\
- Bacilos Gram negativos & \\
Meningitis bacterianas asépticas & Algunas meningitis virales (ocasionalmente) \\
- Mycobacterium tuberculosis & $-\quad$ Virus de la parotiditis \\
- Listeria monocytogenes & $-\quad$ Enterovirus \\
- Borrelia burgdorferi & $-\quad$ Herpesvirus tipo 1 \\
- Brucella melitensis/abortus & $-\quad$ Herpes zóster \\
- Bartonella henselae & $-\quad$ Coriomeningitis linfocítica \\
Meningitis por hongos & Meningitis por treponema \\
- Criptococus sp & $-\quad$ Treponema pallidum \\
& Meningitis por amebas de vida libre \\
& $-\quad$ Naegleria fowleri \\
& Meningitis neoplásicas \\
& Sarcoidosis \\
\hline
\end{tabular}

Valores estimados para un rango de glicemia de $75 \mathrm{mg} / \mathrm{dL}-85 \mathrm{mg} / \mathrm{dL}$ al momento de la PL.

de Lyme $^{7}$ a pesar haber sido considerada en los diagnósticos diferenciales.

En el paciente no se encontraron células neoplásicas en el LCR (examen citológico y citometría de flujo).

Una meningitis por Listeria monocytogenes fue también considerada, aunque esta condición afecta predominantemente a pacientes inmunocomprometidos y embarazadas, pero está documentada en individuos inmunocompetentes ${ }^{5,6}$. El enfermo recibió ampicilina i.v. en altas dosis con una mejoría de la fiebre y cefalea. Tras la suspensión del tratamiento hubo una recaída sintomática. Finalmente, se asoció la fiebre recurrente y el antecedente de un viaje a México un mes antes del inicio de los síntomas, y se realizó una nueva PL que demostró un LCR inflamatorio con persistencia de la hipoglucorraquia. Se solicitó un estudio de ELISA (IgG e IgM) y una prueba de aglutinación para brucela. Ambas fueron positivas: IgG e IgM en la prueba de ELISA y una dilución de 1:320 en la prueba de aglutinación. En este rango, la prueba de aglutinación es comparable a la PCR, considerada el estándar de oro para el diagnóstico ${ }^{8}$. El paciente fue tratado con ceftriaxona $2 \mathrm{~g}$ cada 12
$12 \mathrm{~h}$ i.v. por 15 días y rifampicina oral $600 \mathrm{mg} /$ día por 6 semanas. Estuvo asintomático a partir de la primera semana de tratamiento.

La brucelosis es la zoonosis más frecuente en el mundo ${ }^{9,10}$, con una incidencia estimada en 500.000 casos al año, pero con una gran variabilidad regional $^{9-11}$. El primer caso informado en nuestro país fue en 1930 por Alessandri y González ${ }^{12}$. Olivares y colaboradores ${ }^{13}$ describieron una serie de 13 pacientes diagnosticados con brucelosis en un periodo de 15 años (2000-2016), la mayoría en relación con el consumo de productos lácteos no pasteurizados. Los síntomas predominantes fueron decaimiento y fiebre, sin encontrarse compromiso del $\mathrm{SNC}^{13}$. Se estima que la invasión del SNC tiene una frecuencia entre $2-5 \%$, siendo la meningitis aguda o crónica la complicación neurológica más frecuente $\mathrm{e}^{14-18}$. La meningitis representa entre 17 y $74 \%$ de los casos de neurobrucelosis (NB) informados en la literatura mundial ${ }^{14-18}$. En una de las series más grandes de NB, en un centro de referencia en Kosovo, se describen las características clínicas en 82 pacientes con NB de un total de 648 individuos diagnosticados con brucelosis entre 1991 y $2013^{19}$. De los pacientes con NB, 42\% 
se manifestó con síntomas radiculares, $34 \%$ como meningitis, $12,2 \%$ como meningoencefalitis, $4 \%$ como mielitis transversa y $1 \%$ como un accidente vascular ${ }^{19}$.

En nuestro paciente, que no se encontraba el agente causal de su MA, pero en el contexto de un cuadro meníngeo y febril recurrente, además del antecedente epidemiológico de un viaje a una zona endémica de brucelosis y una hipoglucorraquia persistente algo mayor a los $25 \mathrm{mg} / \mathrm{dL}$, finalmente nos hizo sospechar una NB. Afortunadamente, la NB tiene una buena respuesta al tratamiento y una baja tasa de mortalidad, aunque hay casos en que puede recurriri, ${ }^{9,16}$.

\section{Referencias}

1. Connolly KJ, Hammer SM. The acute aseptic meningitis syndrome. Infect Dis Clin North Am 1990; 4 (4): 599622.

2. Ray P, Badarou-Acossi G, Viallon A, Boutoille D, Arthaud M, Trystram D, et al. Accuracy of the cerebrospinal fluid results to differentiate bacterial from non bacterial meningitis, in case of negative gram-stained smear. Am J Emerg Med 2007; 25 (2): 179-84.

3. Tattevin P, Tchamgoue S, Belem A, Benezit F, Pronier C, Revest M. Aseptic meningitis. Rev Neurol (Paris) 2019; 175 (7-8): 475-80.

4. Chow E, Troy SB. The differential diagnosis of hypoglycorrhachia in adult patients. Am J Med Sci 2014; 348 (3): 186-90.

5. Doolittle BR, Alias A. Application of a prediction rule to discriminate between aseptic and bacterial meningitis in adults. Hosp Pract (1995) 2009; 37 (1): 93-7.

6. Shukla B, Aguilera EA, Salazar L, Wootton SH, Kaewpoowat Q, Hasbun R. Aseptic meningitis in adults and children: Diagnostic and management challenges. J Clin Virol 2017; 94: 110-4.

7. Villagra M, Martínez MJ. [Lyme disease: about an imported case report]. Rev Chilena Infectol 2018; 35 (5): 606-11.

8. Purwar S, Metgud SC, Mutnal MB, Nagamoti MB, Patil CS. Utility of Serological Tests in the Era of Molecular
Testing for Diagnosis of Human Brucellosis in Endemic Area with Limited Resources. J Clin Diagn Res 2016; 10 (2): DC26-9.

9. Pappas G, Akritidis N, Bosilkovski M, Tsianos E. Brucellosis. The New England journal of medicine 2005; 352 (22): 2325-36.

10. Pappas G, Papadimitriou P, Akritidis N, Christou L, Tsianos EV. The new global map of human brucellosis. Lancet Infect Dis 2006; 6 (2): 91-9.

11. Ducrotoy MJ, Ammary K, Ait Lbacha H, Zouagui Z, Mick V, Prevost L, et al. Narrative overview of animal and human brucellosis in Morocco: intensification of livestock production as a driver for emergence? Infect Dis Poverty 2015; 4: 57.

12. Alessandri RH, González AF. Un caso de infección melitocóccica. Rev Med Chile 1931; 59: 7.

13. Olivares R, Vidal P, Sotomayor C, Norambuena M, Luppi M, Silva F, et al. [Brucellosis in Chile: Description of a series of 13 cases]. Rev Chilena Infectol 2017; 34 (3): 243-7.

14. Bouza E, Garcia de la Torre M, Parras F, Guerrero A, Rodriguez-Creixems M, Gobernado J. Brucellar meningitis. Rev Infect Dis 1987; 9 (4): 810-22.

15. Ceran N, Turkoglu R, Erdem I, Inan A, Engin D, Tireli $\mathrm{H}$, et al. Neurobrucellosis: clinical, diagnostic, therapeutic features and outcome. Unusual clinical presentations in an endemic region. Braz J Infect Dis 2011; 15 (1): 52-9.

16. Guven T, Ugurlu K, Ergonul O, Celikbas AK, Gok SE, Comoglu S, et al. Neurobrucellosis: clinical and diagnostic features. Clin Infect Dis 2013; 56 (10): 1407-12.

17. Karsen H, Tekin Koruk S, Duygu F, Yapici K, Kati M. Review of 17 cases of neurobrucellosis: clinical manifestations, diagnosis, and management. Arch Iran Med 2012; 15 (8): 491-4.

18. Turel O, Sanli K, Hatipoglu N, Aydogmus C, Hatipoglu H, Siraneci R. Acute meningoencephalitis due to Brucella: case report and review of neurobrucellosis in children. Turk J Pediatr 2010; 52 (4): 426-9.

19. Dreshaj S, Shala N, Dreshaj G, Ramadani N, Ponosheci A. Clinical Manifestations in 82 Neurobrucellosis Patients from Kosovo. Mater Sociomed 2016; 28 (6): 408-11. 